\title{
NOTE ON CONVEX CURVES ON THE HYPERBOLIC PLANE
}

\author{
L. A. SANTALó
}

1. Introduction. In a previous note [5]( $\left.{ }^{1}\right)$ we have obtained some properties referring to convex curves on the sphere. Following an analogous way our purpose is now to obtain the same properties for convex curves on a surface of constant negative curvature $K=-1$, or, what is equivalent, for convex curves on the hyperbolic plane.

In $\$ \S 6$ and 7 we consider the curves of constant breadth, for which we obtain the formula (7.3) which relates the length $L$ and area $F$ with the breadth $\alpha$.

For the curves which are not of constant breadth the formula (4.5), which contains (7.3) as a particular case, holds. But (4.5) is true only if we suppose that the curve has in all its points geodesic curvature $\kappa_{g}$ grèater than one.

2. Definitions. A closed curve $C$ on a surface of constant negative curvature $K=-1$ is said to be convex when it cannot be cut by any geodesic in more than two points, except that a complete arc of geodesic may belong to the curve. Any closed convex curve $C$ has a finite length $L$ and bounds a finite area $F$. In the following, unless otherwise specified, we shall suppose that $C$ is composed of a finite number of arcs each with continuous geodesic curvature $\kappa_{g}$.

Let $\omega_{i}$ be the exterior angles which these arcs form at the vertices of $C$. Then we have the Gauss-Bonnet formula [3, p. 191],

$$
\int_{C} \kappa_{g} d s+\sum \omega_{i}=2 \pi+F .
$$

If a point $O$ on $C$ is taken as origin, any point $A$ of $C$ can be determined by the length of the arc $O A=s$ or by the angle $\tau$ defined by

$$
\tau=\int_{0}^{s} \kappa_{0} d s+\sum_{s} \omega_{i}
$$

where $\sum_{8} \omega_{i}$ is extended over all the vertices of $C$ contained in the $\operatorname{arc} O A$.

Any geodesic with only one common point or with a complete arc in common with $C$ is called a "geodesic of support" of $C$. In each

Received by the editors November 18, 1944.

${ }^{1}$ Numbers in brackets refer to the references cited at the end of the paper. 
point of $C$ for which there exists a tangent geodesic the geodesic of support coincides with this. Any geodesic which passes through a vertex without crossing $C$ is also a geodesic of support. To any geodesic of support of $C$ corresponds a value of the angle $\tau$ (2.2).

Let $g$ be a geodesic of support of $C$ and let $A$ be its point of support. Let $g^{\prime}$ be the orthogonal geodesic to $g$ at the point $A$ and $g_{1}$ another geodesic of support of $C$ which is also orthogonal to $g^{\prime}$. The curve $C$ will be contained between $g$ and $g_{1}$. If $A^{\prime}$ is the point in which $g_{1}$ cuts $g^{\prime}$, we shall call breadth $\alpha$ of $C$ corresponding to the point $A$ the length of the $\operatorname{arc} A A^{\prime}$ of geodesic $g^{\prime}$. The breadth $\alpha$ is a function of the angle $\tau$ or the arc $s$ corresponding to the point $A$.

3. Closed convex curves with $\kappa_{g}>1$ at any point. Let us suppose that $C$ has $\kappa_{g}>1$ at any point. We shall call pseudospherical osculating circle of $C$ at the point $A$ the limit of the geodesic circle determined by the points $A, A_{1}, A_{2}$ of $C$ when $A_{1}, A_{2} \rightarrow A$.

If we suppose $\kappa_{g}>1$, the radius $R$ of the pseudospherical osculating circle (which we shall call "radius of pseudospherical curvature") has a finite value and is related to the geodesic curvature $\kappa_{\theta}$ by

$$
\kappa_{g}=\operatorname{coth} R \text {. }
$$

This equality is obtained by applying the Gauss-Bonnet formula to a geodesic circle and using the following formulas for its length and area:

$$
L=2 \pi \sinh R, \quad F=2 \pi(\cosh R-1) .
$$

The center $O$ of pseudospherical curvature is the limiting position of the point in which the orthogonal geodesic to $C$ at $A(s)$ cuts the orthogonal geodesic at $A(s+\Delta s)$ when $\Delta s \rightarrow 0$.

The condition $\kappa_{g}>1$, which is equivalent to $R<\infty$, will then be necessary and sufficient for two sufficiently close orthogonal geodesics to $C$ to intersect each other.

Before proceeding it is necessary that we prove the following lemma.

LemMA. Let $g_{1}$ and $g_{2}$ be two geodesics which are orthogonal to the geodesic $g$. Let $M N$ be an arc of the curve $C$ with $\kappa_{0}>1$ at each point and which is tangent to $g_{1}$ and $g_{2}$ at the ends $M, N$ respectively. We affirm that: on the arc $M N$ there is only one point with the property that the orthogonal geodesic to $C$ which passes through it is also orthogonal to $g$.

We suppose the arc $M N$ is composed of a finite number of arcs with continuous geodesic curvature, and a geodesic will be considered 
orthogonal to $M N$ at a corner if it is orthogonal to a geodesic of support through the corner.

Proof. The angle which the geodesics of support of the arc $M N$ form with the orthogonal geodesic to $g$ through their contact points increases from 0 to $\pi$. Hence there is a point at which this angle equals $\pi / 2$. It remains to be proved that there is no other point with this property.

Let us consider the curvilinear coordinate system formed by the orthogonal geodesics to $g$ as curves $v=$ const. and their orthogonal trajectories as curves $u=$ const., $u=0$ being the geodesic $g$. Then the element of length is given by [3, p. 282]

$$
d s^{2}=d u^{2}+\cosh ^{2} u d v^{2} .
$$

If $u=u(s), v=v(s)$ are the equations of the curve $C$, calling $\phi$ the angle which $C$ forms at each point with the corresponding geodesic $v=$ const., we have $\tan \phi=\cosh u v^{\prime} / u^{\prime}$, hence

$$
d \tan \phi / d s=\left[\left(u^{\prime} v^{\prime \prime}-u^{\prime \prime} v^{\prime}\right) \cosh u+u^{\prime 2} v^{\prime} \sinh u\right] u^{\prime-2} .
$$

The geodesic curvature of the curve $u=u(s), v=v(s)$ is given by $[3$, p. 187]

$$
\kappa_{g}=\left(u^{\prime} v^{\prime \prime}-u^{\prime \prime} v^{\prime}\right) \cosh u+2 u^{\prime 2} v^{\prime} \sinh u+v^{\prime 3} \cosh ^{2} u \sinh u .
$$

From this and from (3.3) we deduce

(3.6) $\quad\left(u^{\prime} v^{\prime \prime}-u^{\prime \prime} v^{\prime}\right) \cosh u+u^{\prime 2} v^{\prime} \sinh u=\kappa_{0}-v^{\prime} \sinh u$.

From $u^{\prime 2}+v^{\prime 2} \cosh ^{2} u=1$ and $\cosh ^{2} u-\sinh ^{2} u=1$ we have $v^{\prime 2} \sinh ^{2} u$ $=1-u^{\prime 2}-v^{\prime 2}$, that is, $v^{\prime 2} \sinh ^{2} u \leqq 1$. Hence, from (3.4) and (3.6) under the assumption that $\kappa_{g}>1$, we get (if $u^{\prime} \neq 0$ )

$$
d \tan \phi / d s=\left(\kappa_{0}-v^{\prime} \sinh u\right) u^{\prime-2}>0 .
$$

The angle $\phi$ is then always increasing from $M$ to $N$, that is, from 0 to $\pi$. Consequently at only one point will $\phi=\pi / 2$, which proves our lemma.

4. Principal formula. Let $g$ be a geodesic which cuts the closed convex curve $C$ and let $O$ be a fixed point on the surface with $K=-1$ that contains $C$. Let $w$ be the distance from $O$ to $g$ and $\theta$ the angle which the orthogonal geodesic from $O$ to $g$ makes with a fixed direction at $O$. Then it is known [4, p. 687] that the measure of a set of geodesics is the integral of the expression $d g=\cosh w d \theta d w$ extended to the set. 
Consider the set of "oriented" geodesics which cut $C$; it is known $[4$, p. 691] that

$$
\int_{C, \sigma \neq 0} d g=\int \cosh w d \theta d w=2 L,
$$

where $L$ is the length of $C$.

According to the former lemma if we suppose that $\kappa_{\theta}>1$ in each point of $C$, on each side of $g$ there will be only one point $A$ in which the orthogonal geodesic to $C$ will also be orthogonal to $g$. Consequently the geodesic $g$ can be determined by the point $A$ (that is, by the corresponding value of $s$ or $\tau$ ) and the distance $a$ from $A$ to $g$ (Fig. 1). We wish to express the density $d g=\cosh w d \theta d w$ in terms of $\tau$ and $a$. It is known that the differential expression $d g=\cosh w d \theta d w$

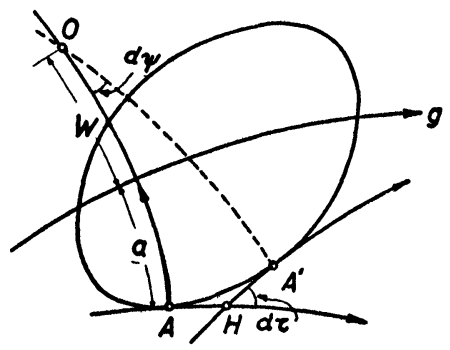

FIG. 1

does not depend on the point $O$ or the direction origin of the angles $\theta$. Consequently we can consider for a moment that the point $O$ is the pseudospheric center of curvature of $C$ at $A$, that is, the point in which the orthogonal geodesic to $C$ at $A(\tau)$ is intersected by the orthogonal geodesic at $A^{\prime}=A(\tau+d \tau)$. Hence $O A=R, w=R-a$. Let $H$ be the point in which the geodesic of support of $C$ at $A$ intersects the geodesic of support at $A^{\prime}$. From the Gauss-Bonnet theorem we deduce that the area of the geodesic quadrilateral $O A H A^{\prime}$ has the value

$$
(\pi / 2+d \tau+\pi / 2+\pi-d \psi)-2 \pi=d \tau-d \psi,
$$

$d \tau$ being the angle which the geodesic of support at $A$ forms with the geodesic of support at $A^{\prime}$ and $d \psi$ the angle $A O A^{\prime}$. But save for infinitesimals of second order the same area equals the area of the sector of geodesic circle $A O A^{\prime}$ which has the value $(\cosh R-1) d \psi$. Consequently

$$
d \tau=\cosh R d \psi \text {. }
$$

Since $R$ is independent of $w$, from $w=R-a, d \theta=d \psi$, and (4.3) we 
deduce

$$
|\partial(a, \tau) / \partial(\theta, w)|=\cosh R \text {. }
$$

Hence $d g=\cosh w d \theta d w=(\cosh (R-a) / \cosh R) d a d \tau$ or, since $d \tau=$ $\kappa_{g} d s$, we have

$$
d g=\cosh a d a d \tau-\sinh a d a d s .
$$

Let us substitute this expression (4.4) in (4.1). For each value of $s$ (or $\tau$ ) the arc $a$ can vary from 0 to the breadth $\alpha$ of $C$ corresponding to the point $s$ (or $\tau$ ). Therefore

$$
\int_{C, g \neq 0} d g=\int_{C} d \tau \int_{0}^{\alpha} \cosh a d a-\int_{C} d s \int_{0}^{\alpha} \sinh a d a
$$

or, in accordance with (4.1),

$$
L=\int_{C} \sinh \alpha d \tau-\int_{C} \cosh \alpha d s .
$$

This is our principal formula from which we wish to obtain some consequences. The formula (4.5) is analogous to that obtained for convex spherical curves in a previous paper [5] and holds for any convex curve with $\kappa_{g}>1$ at each point on a surface of constant negative curvature $K=-1$.

5. Consequences. (a) Let $\Delta$ be the minimum breadth of $C$, that is to say, the minimum value of $\alpha$, and $\delta$ the maximum value of $\alpha$, that is, the diameter of $C$. From (4.5), (2.1), and (2.2) we deduce

$$
\sinh \Delta /(1+\cosh \delta) \leqq L /(2 \pi+F) \leqq \sinh \delta /(1+\cosh \Delta) .
$$

Therefore: on a surface of constant negative curvature $K=-1$, for any convex curve $C$ with $\kappa_{g}>1$ the inequalities (5.1) are verified.

(b) If $C$ has a continuous geodesic curvature, from (4.5) we deduce

$$
\int_{C} \sinh \alpha \kappa_{g} d s-\int_{C}(1+\cosh \alpha) d s=0
$$

or

$$
\int_{C} \cosh \frac{\alpha}{2}\left(\kappa_{g} \sinh \frac{\alpha}{2}-\cosh \frac{\alpha}{2}\right) d s=0 .
$$

From this and according to (3.1), we have: In any closed convex curve $C$ on a surface of constant negative curvature $K=-1$, with continuous goedesic curvature $\kappa_{g}>1$, there are at least two points for which 
the radius $R$ of pseudospherical curvature equals $\alpha / 2$, where $\alpha$ is the breadth of $K$ corresponding to the point considered.

6. Convex curves of constant breadth. In $\$ 2$ we have defined the breadth $\alpha$ of a closed convex curve $C$ on the surface with $K=-1$, corresponding to a point $A$ of $C$. When $\alpha$ is constant, $C$ is called a curve of constant breadth.

The geodesic circles of finite radius $R$ are the first examples of curves of constant breadth $\alpha=2 R$. Another class of curves of constant breadth is the generalization on the surfaces of constant negative curvature of the Reuleaux polygons [2, p. 130].

Let us consider a geodesic circle of radius $R$; we divide it into $2 n+1$ equal parts and through the division points we draw the tangent geodesics. If two consecutive tangents intersect each other we shall have a geodesic regular polygon of an odd number of sides. Taking each vertex as center let us draw the arc of the geodesic circle which joins the two opposite vertices. These arcs form a Reuleaux polygon of $2 n+1$ sides, and it is easily seen that this polygon has constant breadth.

The necessary relation can easily be found between the radius $R$ of the geodesic circle and the number $2 n+1$ of sides so that two consecutives tangent geodesics intersect. Let $A_{i}$ and $A_{i+1}$ be two consecutive division points and $O H$ the geodesic which halves the angle $A_{i} O A_{i+1}$. The condition that the geodesic $O H$ be cut for the tangent geodesic at $A_{i}$ is that the angle $A_{i} O H=\pi / 2 n+1$ be smaller than the angle of parallelism corresponding to the tangent geodesic at $A_{i}$ and to the center $O$, hence $[1$, p. 621$]$

$$
\tan (\pi /(2 n+1))<2 e^{R} /\left(e^{2 R}-1\right) .
$$

That is, to have a Reuleaux polygon of $2 n+1$ sides we must start from a geodesic circle with a radius $R$ which satisfies the inequality

$$
R<\log \frac{1}{\tan (\pi / 2(2 n+1))} .
$$

7. Properties of the convex curves of constant breadth. The convex curves of constant breadth on the surfaces of constant negative curvature (or on the hyperbolic plane) have analogous properties to the curves of constant breadth on the plane. For example it is easily seen that the constant breadth $\alpha$ equals the diameter $\delta$. Therefore if $A$ is a point of $C$ the orthogonal geodesic $g$ to $C$ at $A$ will cut $C$ at the point $A^{\prime}$ and at this point $g$ will also be orthogonal to $C$. The points $A$ and $A^{\prime}$ can be called opposite points. 
We shall prove that if $C$ is of constant breadth any orthogonal geodesic to $C$, say $A A^{\prime}$, will intersect a neighbouring orthogonal geodesic at a point contained in the $\operatorname{arc} A A^{\prime}$.

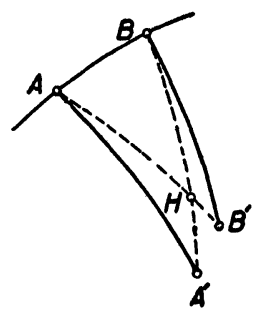

FIG. 2

Let $B \equiv A(\tau+d \tau)$ and $B B^{\prime}$ be the orthogonal geodesic to $C$ at $B$ (Fig. 2). If $B B^{\prime}$ does not intersect the arc $A A^{\prime}$ the point $B^{\prime}$ must have the position indicated in Fig. 2. But in this position we have

$$
A A^{\prime}<A H+H A^{\prime}, \quad B B^{\prime}<B H+H B^{\prime}
$$

and by addition

$$
A A^{\prime}+B B^{\prime}<A B^{\prime}+B A^{\prime} .
$$

Since $C$ is of constant breadth we have $A A^{\prime}=B B^{\prime}>A B^{\prime}, B A^{\prime}$ and therefore $A A^{\prime}+B B^{\prime}>A B^{\prime}+B A^{\prime}$ which gives a contradiction with (7.2).

From this we deduce that the pseudospherical radius of curvature $R$ is always finite and not greater than $\alpha$. Moreover since the orthogonal geodesics at $A$ and $B$ are also orthogonal geodesics at $A^{\prime}$ and $B^{\prime}$ the pseudospherical center of curvature will be the same at $A$ and $A^{\prime}$. Hence: the sum of the pseudospherical radii of curvature corresponding to opposite points equals the breadth $\alpha$.

Since $R<\infty$, we shall have $\kappa_{0}>1$; therefore we can apply the formula (4.5) which gives, according to (2.1),

that is,

$$
L=(2 \pi+F) \sinh \alpha-L \cosh \alpha,
$$

$$
L=(2 \pi+F) \tanh (\alpha / 2) .
$$

We conclude the following theorem: For any convex curve of constant breadth on the surface of constant negative curvature $K=-1$, the equation (7.3) holds.

If $K=-1 / a^{2}$ instead of $-1,(7.3)$ gives

$$
L / a=\left(2 \pi+F / a^{2}\right) \tanh (\alpha / 2 a) .
$$


Muliplying both sides by $a$ and letting $a \rightarrow \infty$. we find

$$
L=\pi \alpha,
$$

which is a well known relation between the length and breadth of the curves of constant breadth on the plane [2, p. 131].

\section{REFERENCES}

1. L. Bianchi, Lezioni di geometria differenziale, 3d ed., vol. 1, Bologna, 1927.

2. T. Bonnesen, and W. Fenchel, Theorie der konvexen Körper, Ergebnisse der Mathematik und ihrer Grenzgebiete, Berlin, 1934.

3. L. P. Eisenhart, An introduction to differential geometry, Princeton, 1940.

4. L. A. Santal6, Integral geometry on surfaces of constant negative curvature, Duke Math. J. vol. 10 (1943).

5. - Note on convex spherical curves, Bull. Amer. Math. Soc. vol. 50 (1944).

Universidad NACIONAL DEL Litoral, Rosario 